\title{
Observation of Localized Electromagnetic Waves in Three-Dimensional Networks Of Waveguides
}

\author{
Z. Q. Zhang, C. C. Wong, K. K. Fung, Y. L. Ho, W. L. Chan, S. C. Kan, T. L. Chan, and N. Cheung \\ Department of Physics, Hong Kong University of Science and Technology, \\ Clear Water Bay, Kowloon, Hong Kong, China
}

(Received 13 May 1998; revised manuscript received 1 October 1998)

\begin{abstract}
We have directly observed the Anderson localized wave functions in three dimensions in a new class of photonic band gap systems. Such systems are networks made of one-dimensional waveguides. By adopting a simple scattering geometry in a unit cell, we are able to obtain large photonic band gaps. In the presence of defects or randomness, we have systematically studied the structures of transmission and the localized wave functions inside a gap. The effects due to absorption are investigated. Excellent quantitative agreements between theory and experiments have been obtained. [S0031-9007(98)07988-5]
\end{abstract}

PACS numbers: $41.20 . \mathrm{Jb}$

In the past decade, the localization of classical waves in random media has been under intensive studies [1]. Unlike electrons, the localization of classical waves is purely a result of multiple scatterings in a random environment and free from the complications arising from interaction effects. However, due to the Rayleigh scattering at low frequencies, it is more difficult to localize classical waves than to localize electrons. In 3D, waves can be localized only in certain windows in the intermediate frequency range with a minimum dielectric contrast [2]. It has been suggested that waves are more easily localized inside a gap or pseudogap of a photonic band gap (PBG) material [3]. The PBG material in its own right is of great interest and has important implications in both fundamental science and technological applications [4]. The localization of electromagnetic waves has been observed in 1D and 2D PBG materials [5,6]. For 3D systems, efforts have been focused only on wave localization in random media. The effects arising from wave localization have been reported in microwave experiments [7]. Nevertheless, a direct interpretation of localization was complicated by the presence of large absorption. Very recently, direct evidence of light localization has been reported in strong scattering media of semiconductor powders based on the size dependence of the transmission coefficient [8]. These important developments lead us to question if one can directly observe Anderson localized wave functions in 3D. For this purpose, like earlier investigations in 2D [6], we need to investigate the strongly localized states inside the gap of a PBG system, where the localization length is short. In addition, a direct measurement of the 3D wave function should be allowed in such systems.

To meet these two requirements, we propose a new class of PBG systems here. Such systems are networks connected by segments of 1D waveguides [9]. There are two important advantages in such systems. First, strong scattering can be easily introduced in a unit cell to produce large full gaps in any dimension. Thus, unlike usual PBG systems, our systems do not require a material with a large dielectric constant. Second, the wave function at each node is physically accessible so that 3D localized wave functions can be probed.

In our study, the coaxial cable was adopted as the 1D waveguide. The 3D network considered was in a diamond structure. The structure can also be constructed as alternating layers of $A$ and $B$ types as shown in Fig. 1, where every node of the $A$ layer is connected to the corresponding node in the $B$ layer. To enhance the scattering at nodes, we replace all vertical segments in each layer by loops, as indicated by the heavy lines. By including these loops, we are able to produce large full gaps in all dimensions.

This network system can be described by a set of network equations. The propagation of waves in a segment obeys the wave equation $\partial^{2} \varphi / \partial s^{2}=\left(\varepsilon \omega^{2} / c_{o}^{2}\right) \varphi$, where $\omega=2 \pi f$ and $c_{o}$ are, respectively, the wave frequency and speed in the vacuum. For a coaxial cable, $\varphi$ denotes the voltage waves and $\varepsilon=\varepsilon^{\prime}+i \varepsilon^{\prime \prime}$ is the relative permittivity of the dielectric medium. Because of the continuity of wave function and conservation of flux at each node, the wave function at node $i$ satisfies [9]

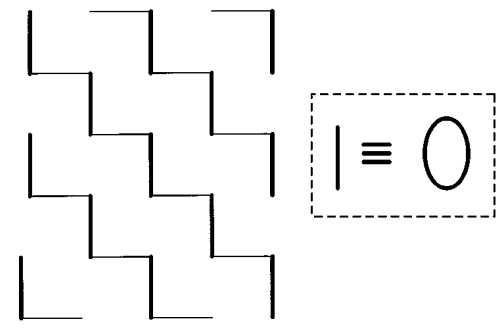

A

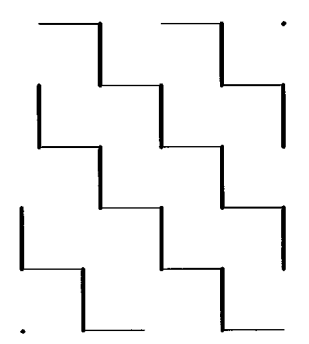

B
FIG. 1. The 3D network in a diamond structure with alternating layers of $A$ and $B$. Inset: Each heavy vertical line denotes a loop geometry. 


$$
\begin{aligned}
&-\varphi_{i} \sum_{j}\left[\sum_{m=1}^{n(i j)} \operatorname{coth}\left(z \ell_{i j}^{(m)}\right)\right] \\
&+\sum_{j}\left[\sum_{m=1}^{n(i j)} \frac{1}{\sinh \left(z \ell_{i j}^{(m)}\right)}\right] \varphi_{j}=0,
\end{aligned}
$$

where $n(i j)$ denotes the number of segments connecting nodes $i$ and $j, \ell_{i j}^{(m)}$ is the length of the $m$ th segment, and the summation of $j$ is over all of the nodes linked directly to $i$. In the case where $\varepsilon^{\prime \prime} \ll \varepsilon^{\prime}, z$ has the simple form $z \cong i k-1 / 2 \ell$ with $k=\omega \sqrt{\varepsilon^{\prime}} / c_{o}$ and the absorption length $\ell=\varepsilon^{\prime} / k \varepsilon^{\prime \prime}$. For a periodic network, band structures can be obtained by solving Eq. (1) in a unit cell.

Experimentally, the coaxial cables we used were the RG58A/U type. To probe the system, a HP8648C signal generator was connected at some node on one side of the network and a HP8563E spectrum analyzer was connected at some node on the opposite side of the network to measure the transmission spectrum $T(f)$. Theoretically, the transmission coefficient is calculated by solving Eq. (1) with the presence of both source and receiver terms [9]. The absorption length of the cable was obtained from the attenuation specification data supplied by the manufacturer, i.e., $\ell \cong 1702 f^{-0.59}$, where $\ell$ is in units of $\mathrm{m}$. The extra length in each segment due to the presence of connectors should also be included in $\ell_{i j}^{(m)}$ of Eq. (1). To include the additional dissipation due to connectors, we add a constant $\gamma$ to the terms $z \ell_{i j}^{(m)}$ in Eq. (1). By fitting the calculated transmission coefficient against the measured data in a single loop as shown in the inset of Fig. 1, we found $\varepsilon^{\prime} \cong 2.32$ and $\gamma \cong-0.0075$.

The experimental network we constructed contained five layers in the $z$ direction in the order $A B A B A$ with five nodes in the $x$ direction and six nodes in the $y$ direction in each layer, as shown in Fig. 1 . We use $(5 \times 6 \times 5)$ to describe the size of the network. The two lengths chosen for the loops were 0.5 and $2 \mathrm{~m}$. For all other single segments, the length was $0.5 \mathrm{~m}$. If we denote the node at the lower-left corner of the first layer by $(x, y, z)=$ $(1,1,1)$, the wave was injected from the node $(1,4,3)$ and the transmission was measured at node $(5,3,3)$. For an ordered network, the measured $T(f)$ is plotted by circles in Fig. 2(a). Theoretically, we have calculated $T(f)$ in the presence of dissipation. The result is plotted by a solid curve in Fig. 2(a). Excellent quantitative agreement between theory and experiment is evident. From $T(f)$, we found a wide gap from about 41 to $60 \mathrm{MHz}$ with a peak at about $52 \mathrm{MHz}$. The existence of this gap is independent of the input and output positions, which is the signature of a full gap. To confirm this, we have performed band structure calculations. A full gap from 44 to $59 \mathrm{MHz}$ has been found. The states which appeared in the frequency windows $(41,44)$ and $(59,60) \mathrm{MHz}$ were found to have Bloch wavelengths larger than the sample

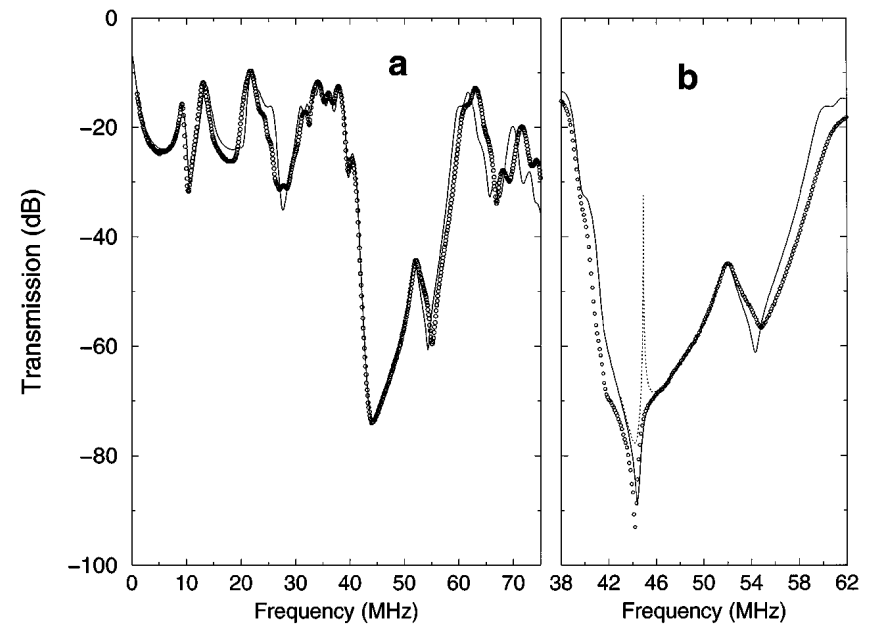

FIG. 2. Transmission coefficient. (a) For an ordered network, the circles and solid curve are the measured and calculated results, respectively. (b) For the defect described in the text, the circles and solid curve are the measured and calculated results, respectively. The dotted curve is the calculated result in the absence of dissipation.

size and, therefore, could not be excited. From the analysis of the scattered wave functions in the absence of dissipation, we found that the peak at about $52 \mathrm{MHz}$ represented surface states.

Before considering random networks, we present the results of a defect state first. There are various ways to introduce a defect into an otherwise ordered network. For instance, if we change one of the two lengths of the loop at the center of the middle layer $(A)$ from 2 to $1.6 \mathrm{~m}$, the measured and calculated $T(f)$ are plotted by the circles and solid curve in Fig. 2(b), respectively. The defect state is indicated by a cusp at $45.0 \mathrm{MHz}$, which is confirmed by a sharp peak in the calculated $T(f)$ in the absence of dissipation (the dotted curve). The location of this defect frequency coincides with that of the cusp in the presence of dissipation. Thus, dissipation reduces the height of a peak drastically and it can even be changed to a cusp.

In the absence of dissipation, a localized wave function $\varphi_{i}$ can be obtained from the scattered wave function in a transmission measurement at the resonant frequency. However, due to dissipation, the large reduction in the transmission coefficient makes the intensity of the scattered wave function decay exponentially away from the input surface. This decay masks the localized nature of the wave function. Nevertheless, the explicit profile of a localized wave function can be retrieved by using wave injection at the center of the localized state [6].

By injecting the wave from one of the defect nodes at $45.0 \mathrm{MHz}$, we measured the voltage at each node. The corresponding intensity map $\left|\varphi_{i}^{\mathrm{mp}}\right|^{2}$ is plotted in Fig. 3(a) for each layer, where the superscript "mp" denotes the measured wave function from injection at the peak position. Here we have normalized the intensity by 
(a)

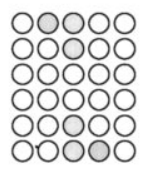

1

(b)

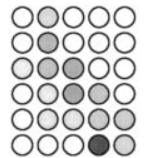

(c)

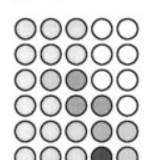

00000

(d)
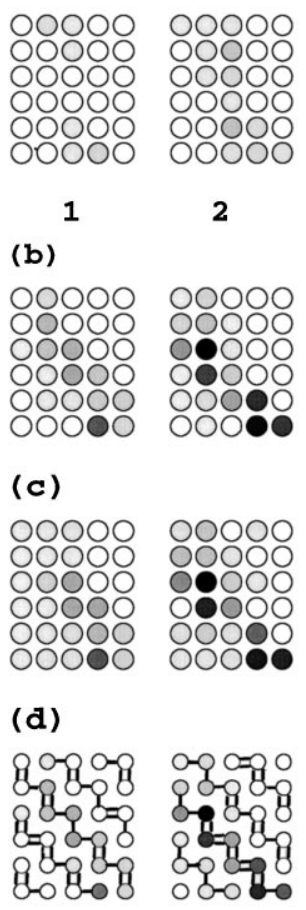

2
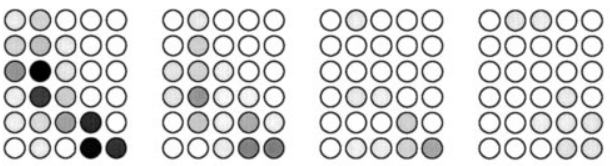

(0000
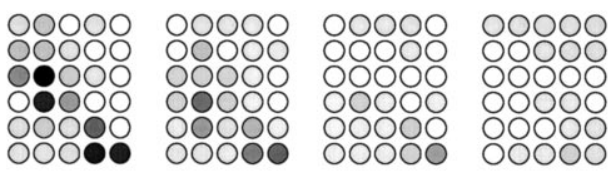

(e)

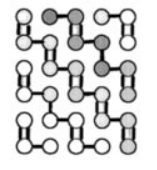

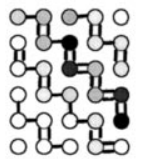
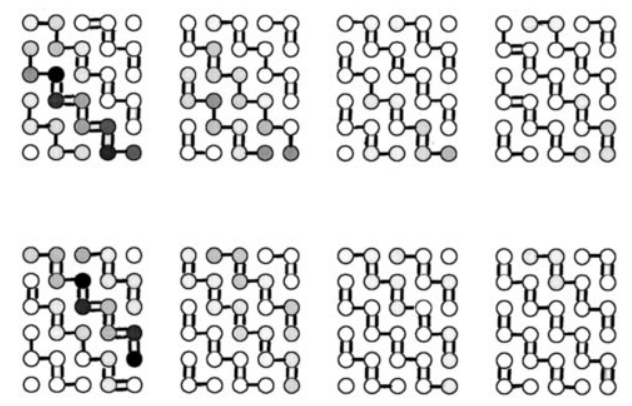

0
FIG. 3. (a) The measured intensity map of a defect state at $f \cong 45.0 \mathrm{MHz}$ shown in Fig. 2. (b) $-(\mathrm{d})$ : The Anderson localized state at $f \cong 46.66 \mathrm{MHz}$ shown in Fig. 4(a). (b) denotes the result obtained from the scattered wave function in the absence of dissipation. (c) and (d) are, respectively, the measured and calculated results by using injection at the peak position in the presence of dissipation. (e) is the intensity map of a multidefect state at $f \cong 46.77$ described in the text. The double and single lines in (d) and (e) denote the configuration of the loops and single segments in each respective network.

choosing the peak value as unity. The relative intensity at each node is represented by the degree of darkness in the corresponding circle. A sharply localized state centered at the defect nodes is clearly seen. We found excellent agreement between the measured $\left|\varphi_{i}^{\mathrm{mp}}\right|^{2}$ and the theoretical $\left|\varphi_{i}\right|^{2}$. A similar defect wave function has also been obtained by replacing a loop in an ordered network with a single segment. The defect mode in such systems provides a new kind of microcavity and may be useful in the applications of microcavity devices [10].

It should be pointed out that in the frequency range of interest here, i.e., $f=40-60 \mathrm{MHz}$, the absorption length is on the order of $150 \mathrm{~m}$. This is much larger than all of the localization lengths presented in this work. The effects due to absorption on the localized wave functions can, therefore, be ignored.

In order to study the Anderson localized states inside the gap, we introduced randomness into the network by randomizing the position of the loops. Starting from an ordered network shown in Fig. 1, we have randomly replaced $x$ percent of single segments in each layer by loops and $x$ percent of loops by single segments. Thus, $x$ measures the amount of disorder in the system. The maximum disorder occurs when $x=50$. The introduction of randomness creates many states inside the gap. The number is roughly equal to the number of defects introduced. In order to measure a single Anderson localized wave function in the presence of dissipation, the value of $x$ cannot be too large. Two nearby states will mix if their frequency separation becomes comparable to the absorption width, which is about $\Delta f \approx c_{o} / 4 \pi \ell \sqrt{\varepsilon^{\prime}}=0.1 \mathrm{MHz}$ in the region of interest here. We have chosen $x=20$, which produces, on average, 25 states inside the gap with an average separation of about $0.8 \mathrm{MHz}$.

To avoid the effects due to surface states, we have focused our study in the region from 38 to $48 \mathrm{MHz}$. The measured and calculated $T(f)$ of a particular configuration are plotted in Fig. 4(a) by the circles and solid curve, respectively. The calculated $T(f)$ without dissipation is plotted by a dotted curve. Each sharp peak inside the gap represents a localized state. To study the wave function, we pick the most isolated state at $46.66 \mathrm{MHz}$ as marked by the double arrows. The corresponding scattered wave function gives the Anderson localized wave function $\varphi_{i}$. We have plotted its intensity map in Fig. 3(b). The wave function is peaked at $(2,4,2)$. By injecting the wave at this position at $46.66 \mathrm{MHz}$, we measured $\left|\varphi_{i}^{\mathrm{mp}}\right|$. In Figs. 3(c) and 3(d), we have plotted the measured $\left|\varphi_{i}^{\mathrm{mp}}\right|^{2}$ and calculated $\left|\varphi_{i}^{\mathrm{cp}}\right|^{2}$. The superscript "cp" denotes the calculated wave function with wave injection at the peak position in the presence of dissipation. We found excellent agreements among Figs. 3(b)-3(d).

In Fig. 3(d), we have also drawn the network configuration by attaching single or double lines between two nodes to denote single segments or loops, respectively. By correlating this configuration with the intensity map, we can always find some defect close to the position where $\left|\varphi_{i}\right|^{2}$ is large. However, there is an important difference between a defect or multidefect state and an Anderson localized state. A defect or multidefect state is created by a defect or a few defects in an otherwise ordered environment. Compared to such states at the similar frequency, an Anderson localized state has a longer decay length due to less effective interference effects in localizing the wave function under a random environment. To show this, we consider a network with defects appearing only in the first two layers. For the configuration shown in Fig. 3(e), the intensity map of a multidefect state with frequency at $46.77 \mathrm{MHz}$ is also shown. Like the Anderson localized state shown in Figs. 3(b)-3(d), 

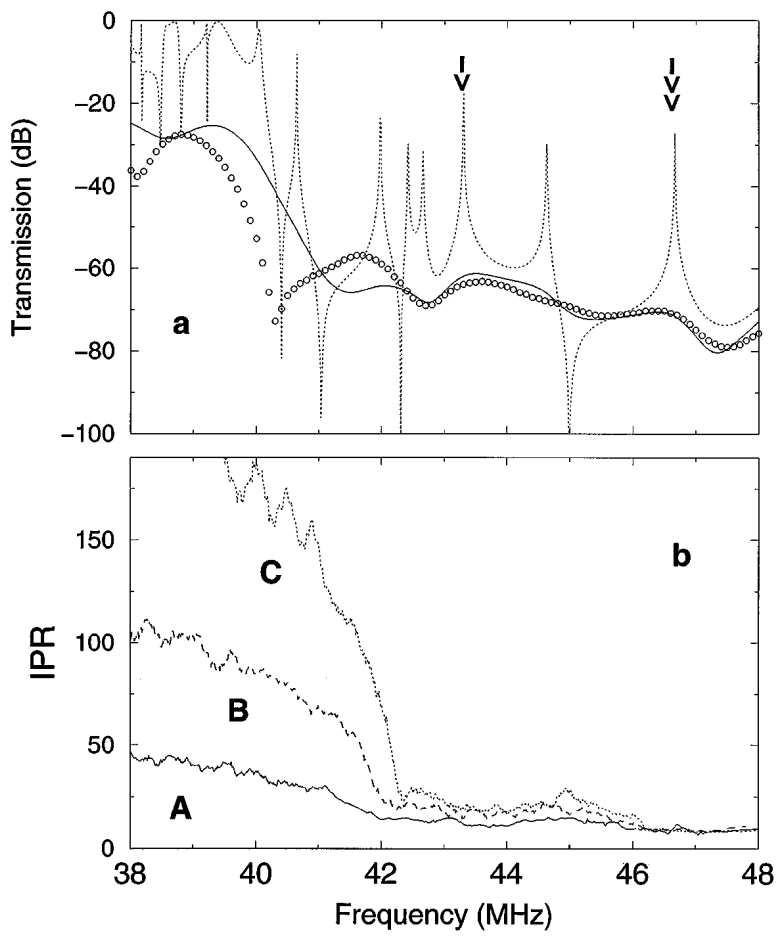

FIG. 4. (a) Transmission coefficient for a random network described in Fig. 3(d). The circles and solid curve are the measured and calculated results. The dotted curve is the calculated result in the absence of dissipation. (b) The inverse participation ratio is plotted as a function of frequency for three sample sizes: $(5 \times 6 \times 5),(7 \times 8 \times 7)$, and $(9 \times 10 \times 9)$, denoted by $A, B$, and $C$, respectively.

the intensity is also concentrated in the second layer with the similar local configuration near the peak positions. Here we would like to study how the layer intensity decays from the second to the fifth layer in both cases. After summing the total intensity in each layer, we found the decay lengths to be 0.52 and 0.76 for the multidefect and Anderson localized states, respectively. For the single defect state shown in Fig. 3(a), the decay length was found to be 0.48 . These numbers clearly show the distinction between a defect or multidefect state and an Anderson localized state. To better understand the nature of the localized state we have observed, we have also calculated the center positions and volumes occupied by all localized states shown in Fig. 4(a) by calculating the mean $\left(x_{c}, y_{c}, z_{c}\right)$ and standard $\left(\sigma_{x}, \sigma_{y}, \sigma_{z}\right)$ deviation of each state. The volume is estimated as $v \approx 8 \sigma_{x} \sigma_{y} \sigma_{z}$. For the state at $46.66 \mathrm{MHz}$, we found $\left(x_{c}, y_{c}, z_{c}\right) \approx(3.3,2.4,2.1)$ and $\left(\sigma_{x}, \sigma_{y}, \sigma_{z}\right) \approx(1.2,1.4,0.7)$. The latter numbers give $v \approx 9.4$ and an average linear size of 2.1. The other nearest center comes from the state at $43.29 \mathrm{MHz}$ [marked by an arrow in Fig. 4(a)] with $\left(x_{c}, y_{c}, z_{c}\right) \approx$ $(3.1,2.4,2.0)$. The separation distance between these two centers is about 0.26 , which is much smaller than the linear size of the state. Also, the average volume occupied by the localized states shown in Fig. 4(a) is about 9.2. This number is larger than the average volume occupied by a defect in the network, i.e., 3.6. All of these numbers indicate that the state we have observed is a single Anderson localized state arising from the multiple scattering among defects in a random environment.

Finally, we investigate the localization characteristics using infinite-size scaling. We use the inverse participation ratio IPR $=\left(\sum_{i}|\varphi|^{2}\right)^{2} / \sum_{i}\left|\varphi_{i}\right|^{4}$ as a measure of the spatial extension of a state [11]. For an extended state across a sample of $N$ nodes, IPR is on the order of $N$ and increases with sample size. For a localized state, IPR is much smaller than $N$ and is independent of sample size. In Fig. 4(b), we plot the averaged IPR as a function of frequency for three sample sizes, i.e., $(5 \times 6 \times 5),(7 \times 8 \times 7)$, and $(9 \times 10 \times 9)$. In the region of 36 to $48 \mathrm{MHz}, 3000,500$, and 100 configurations were taken, respectively. Those states with peak intensities located on the surface were not included in the average. For frequencies near the band edge, IPR is large and increases with size. As frequencies move into the gap, the states become more localized with a smaller IPR and less size dependence. When the frequency falls in the region 46-48 MHz, IPR becomes size independent. This shows that the Anderson localized state we have observed at $46.66 \mathrm{MHz}$ is indeed well localized in our experimental network. It is interesting to point out that, in this frequency region, the average IPR increases with randomness. Its average value increases from IPR $\approx 9$ at $x=20$ to IPR $\approx$ 12 at $x=50$ due to the increase of density of states.

The authors thank P. Sheng, C. T. Chan, and B. F. Zhu for useful discussions. This work was supported by Hong Kong RGC Grant No. HKUST 6137/97P.

[1] See, for example, Scattering and Localization of Classical Waves in Random Media, edited by P. Sheng (World Scientific, Singapore, 1990).

[2] P. Sheng and Z. Q. Zhang, Phys. Rev. Lett. 57, 1879 (1986).

[3] S. John, Phys. Rev. Lett. 58, 2486 (1987).

[4] For example, Photonic Band Gap Materials, edited by C. M. Soukoulis (Kluwer Academic Publishers, Dordrecht, The Netherlands, 1995).

[5] D. Z. Zhang, W. Wu, Y. L. Zhang, L. Li, B. Y. Cheng, and G. Z. Yang, Phys. Rev. B 50, 9810 (1994).

[6] S. L. McCall, P. M. Platzman, R. Dalichaouch, D. Smith, and S. Schultz, Phys. Rev. Lett. 67, 2017 (1991); Nature (London) 354, 53 (1991).

[7] A.Z. Genack and N. Garcia, Phys. Rev. Lett. 66, 2064 (1991); 66, 1850 (1991).

[8] D. S. Wiersma, P. Bartolini, Ad. Lagendijk, and R. Righini, Nature (London) 390, 671 (1997).

[9] Z. Q. Zhang and P. Sheng, Phys. Rev. B 49, 83 (1994).

[10] E. Yablonovitch, T. J. Gmitter, R. D. Meade, A. M. Rappe, K. D. Brommer, and J.D. Joannopulos, Phys. Rev. Lett. 67, 3380 (1991).

[11] J. T. Edwards and D. J. Thouless, J. Phys. C 5, 807 (1972). 